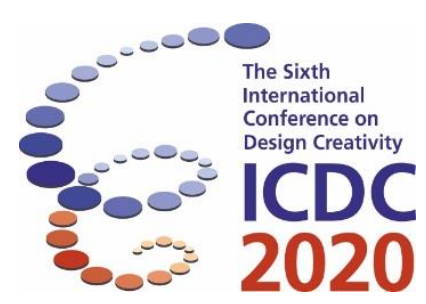

\title{
A Topological Variation-Oriented Approach for Enhancing Creativity in Product Design Education
}

\author{
Fei Fei ${ }^{1}$, Yukari Nagai ${ }^{2}$ and Yang $\mathrm{Yu}^{2,1}$ \\ ${ }^{1}$ School of Art and Design, Dalian Polytechnic University, Dalian, China \\ ${ }^{2}$ School of Knowledge Science, Japan advanced Institute of Science and Technology, Nomi, \\ Japan
}

\begin{abstract}
This study introduces an approach aimed at promoting the effect of product design creativity by using topological properties variations. The topological variation-oriented approach for creativity (TVC) generation showed as a visualized sheet and presented the process of products acting on human. The action process was depicted through four territories on the sheet: human, product, interaction, and experience. Each field is distributed in a vertical direction from left to right in the logical order of human, product, interaction, and experience. Each field is divided into several elements, and corresponding elements of each field are arranged in a horizontal direction. When topological variation occurs on the sheet, there will be some new relationship between elements, and it is very possible to produce new products. Participants use this approach to obtain creative ideas and novel product form. It has strong potential to attract people's attention.
\end{abstract}

Keywords: topological perception; product shape; topological properties variations; creativity; product design education

\section{Introduction}

The appearance of a product is usually the first step in the interaction process of consumer and product (Hollins \& Pugh, 1990). Designers often focus on designing products with novel appearances because these appearances attract attention (Hekkert et al., 2003). Consumers evolved prototypes of product categories after they experienced many different products. Consumers perceive the innovations of products through the outer forms that synchronize with underlying technological updates (Rindova \& Petkova, 2007). Therefore, designers attach great importance to the expression of product innovation from form innovation. More importantly, consumers are more likely to perceive and accept product form innovation. The attractive innovation of the product is reflected in the form and can be explained from the topological variations (Fei \& Nagai, 2019). This paper contributes to research on a creative thinking approach to designing a novel product and the original interaction between human and product. It presents a visualizing sheet of plain paper - showing the activity that people's thinking undergoes during topological variations. Human, product, interaction, and experience are the four territories represented in the visualization column and are broken down as follows: arranged in rows according to each section of the human body corresponding to the sections of product, the interactions of the corresponding human body sections and products, and the user experience of these sections. Several nodes distribute in each domain and every node present as disks visualized elements. The dotted lines present the relationships of the nodes. The topological variations in visual perception (holes, connection 
and inside/outside), the ring present hole, the dotted line connecting a new disk from one present connection, one disk goes into/out another disk present inside/outside, Table 1 . This study selected a bicycle, a mechanical pen, an electric fan, and a mobile phone to elaborate which shapes of these products make topological variations. Human not only generate the new visual perception to these products, but also the products have created a new functional mechanism, and the interaction appears between persons and products in a completely new way at the same time. The prototype of TVC, show in Figure 1.

Table 1. Three visualized symbols of topological properties in two-dimension

\begin{tabular}{|c|c|c|c|}
\hline Prototype & Element & Connectivity & Inside/Outside \\
\hline Visualized symbol & & & \\
\hline Topological variations & Hole & New Connectivity & Outside/Inside \\
\hline Visualized symbol & & & \\
& & & \\
\hline
\end{tabular}

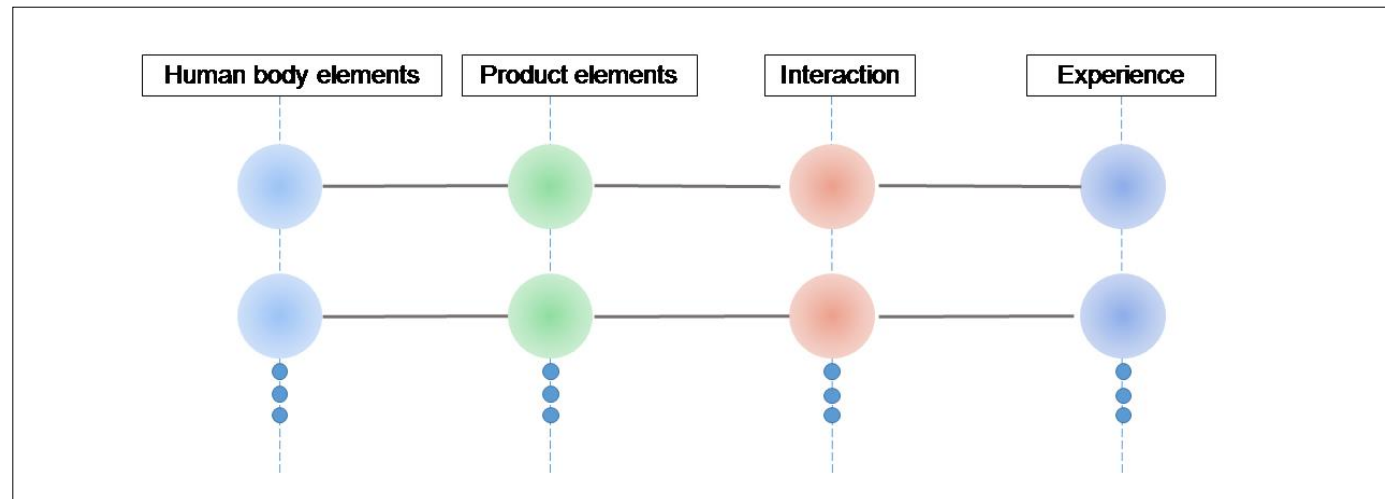

Figure 1. The prototype of the topological variations — oriented creativity generation (TVC)

\section{Background}

Topology is a subject that studies some properties of geometric figures or spaces that remain unchanged after changing shape continuously (Rashevsky, 1954). Topology has nothing to do with the measurement properties and quantity of the object of studies, such as length, size, area, and volume (Darke, I., 1982). It only considers the position relationship between objects without considering their shape and size (Munkres, 2014). Under topological transformations, topological properties such as connectivity, the number of holes, and the inside/outside relationship remain invariant (Chen, 2005). In contrast, symmetry, orientation, size, parallelism, and collinearity are properties of Euclidean geometry. As mentioned above, it has been shown that people can recognize topological differences faster than Euclidean geometric differences. In addition, perception proceeds from local elements taken as features to their integration into a whole (Treisman, 1980), whereas in early holistic registration, perception proceeds from global properties of a visual scene to the analysis of local features (Navon, 1977). Consider the case of topological differences being more discriminable than topologically equivalent objects despite their differences in local features (Chen, 1982). Early topological perception precedes the perception of local features, and global topological variations can receive more attention than local geometric differences. In the aspect of product design, the appearance variants of a product category bear a degree of resemblance in their design features. After repeatedly seeing similar design features in different product variants, these design features are included in consumers' prototype. The prototype 
became a schema in the mind, which possesses the average value of the design features of that category (Ruth \& Jan, 2012). It can be seen that keep the design features without changing the schema cannot get rid of the constraints of product categories. TVC can change the design feature can designers create new schema and create fundamental innovation.

\section{Topological Variations-Oriented Approach for Creativity Generation}

We take a bicycle, mechanical pencil, electric fan, and mobile phone as examples in this study and find that the topological variations of the product shape are closely related to the radical innovation of the structure in the four products.
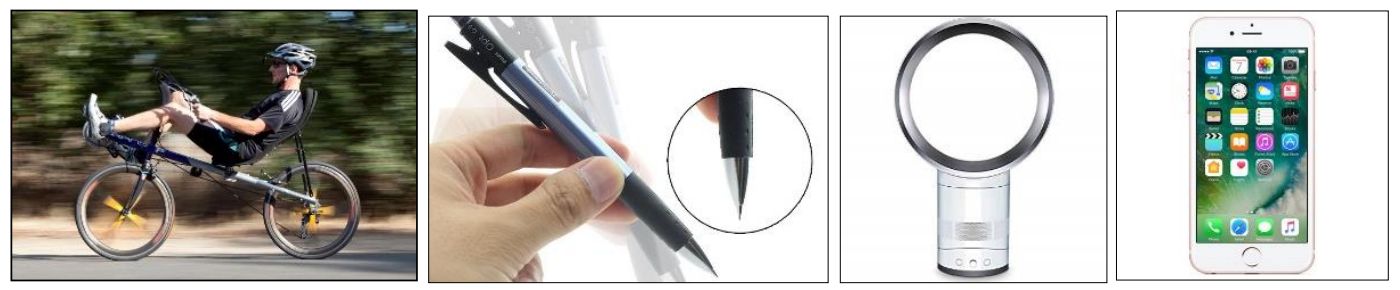

Figure 2. The four typical cases

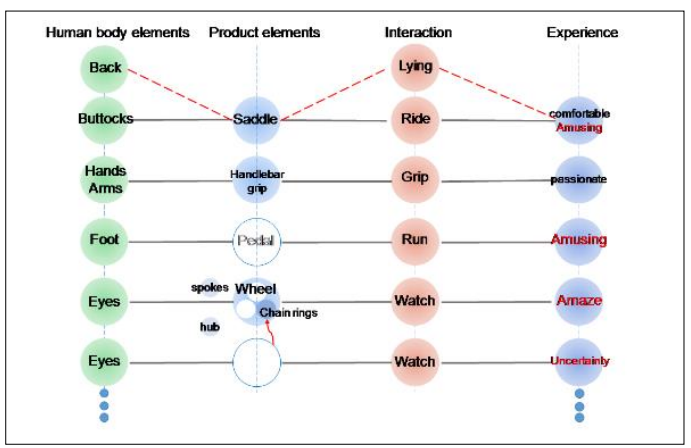

Figure 3. TVC of Back-bicycle

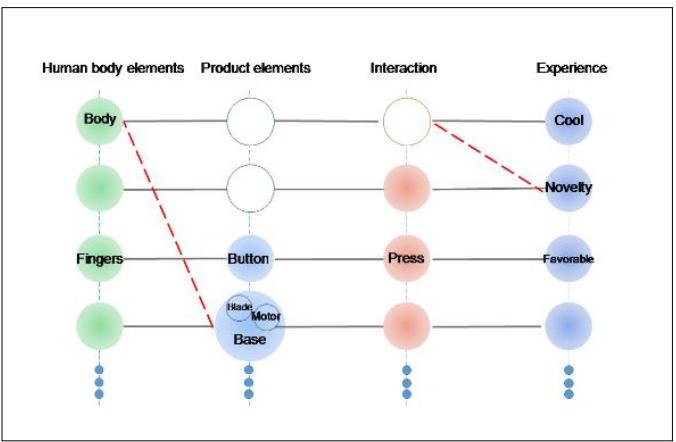

Figure 5. TVC of Bladeless fans

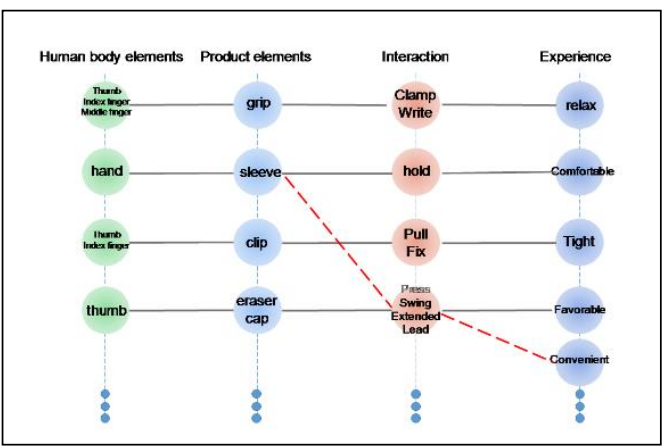

Figure 4. TVC of Mechanical pencil

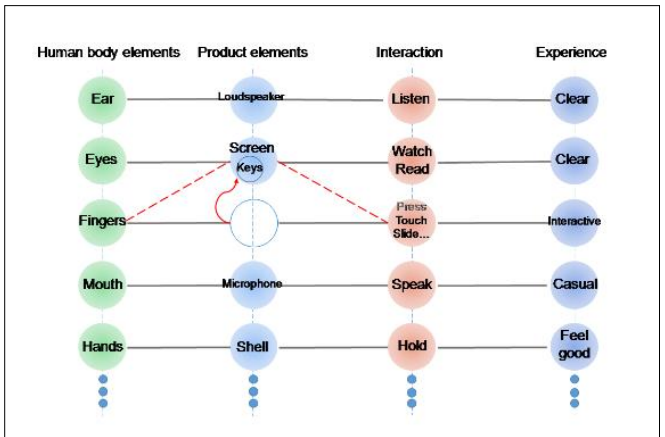

Figure 6. TVC of Smartphone

The bicycle as an example in the experiment about visual attention, it was verified a typical product in which the innovation come from topological variations. The corresponding TVC is shown in Figure 3. The topological variations is built between saddle and back, the user has a musing experiment simultaneously. This is also a very interesting experience.

We found that the difference belongs to topological variant in the Mechanical pencil. The most prominent innovation is that lead can be extended automatically, not by thumb pressing eraser cap, but by wrist shaking. Namely, the logical order of row, eraser cap-thumb-press-extending lead (with changing the gesture of hand). We change two relationships of the three typical ones, i.e. sleeve-handshake and extending lead (keep the gesture of hand), Figure 4. 
In terms of the topological variant in visual perception, if we change the disk into the ring, or take the blades set out of the disk set, and the people still feel cool the flow air taking. Under the condition, we must find a solution that a fan blows air from a ring with no external blades. Its blades are hidden in its base. Thin high-velocity smooth airflow blows from the hole, Figure 5.

Figure 6 shows that the screen departs the keys in the mobile phone, but the smartphones were born until the keys to go into the screen or become a hole. The physical keys disappear and present in the form of virtual ones which essence is software program. Changing the traditional interaction between mobile phone and users into the interface is built around the multi-touch screen device, including a virtual keyboard. Such a major innovation is manifested in the TVC as a topological variant, that is, the set of physical keys can be regarded as an empty set, represented as a ring, or as a set of physical keys entering the screen set.

\section{Experiment design}

The objective of the experiment is to use topological variations to represent a visualized sheet, using it as a tool to stimulate product design creativity of the students who study product design. The creativity of students can be improved by using this visualized sheet.

In the first experiment, recruiting 61 participants ( 31 female; 30 male), 48 juniors and 13 postgraduates majoring in product design. After three years of specialties study, students have a certain degree of product design expertise and skills. The design topic is to design two new chairs. Each person sends out two A3 drawings and carries out a creative design on each paper. Each person produces two sets of design plans. Pencil sketch with 45 degree perspective, normal visual height, 200 words of text description, 90 minutes. Participants are asked to not refer to existing designs, but to their own creativity entirely. Students themselves choose one of the more satisfactory schemes to submit as a backup for the next stage of the experiment. Each design scheme hides the author's personal information, which is represented by numbers 101 to 161 . After four days, during the period, the participants did not receive any other new learning and creative methods, so the improvement of the creative ability of the participants can only be from TVC. The 61 participants were congregated to begin the second stage of the experiment. The purpose of the interval of four days is to minimize the interference between the two chair designs. First, the instructor explained the samples of the above four products of TVC and the three kinds of topological variations to the participants in 30 minutes. The following is the chair's TVC model as a standard reference for the experiment (Figures 7 and 8).

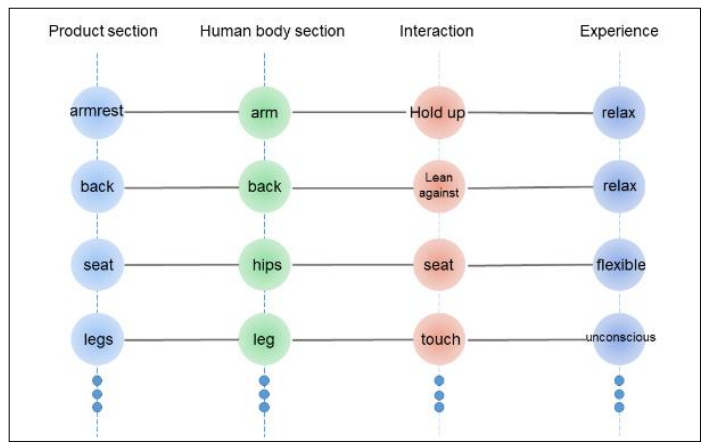

Figure 7 TVC prototypes Chair

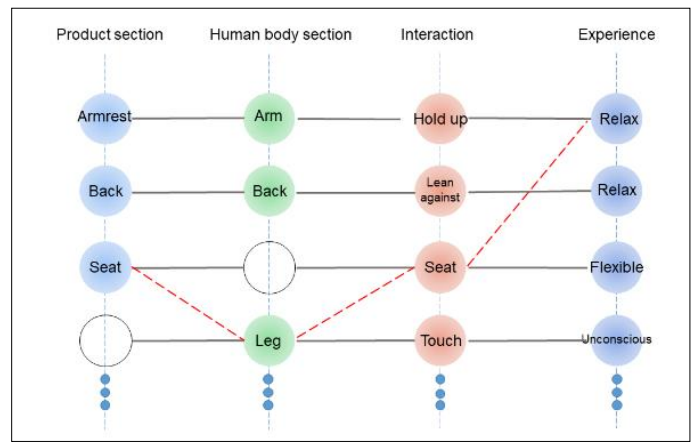

Figure 8 TVC Design Chair

The third stage of the experiment was to organize 45 other product design students to score the two designs, using the 7-point Likert scale. Because this stage focused on the evaluation of the design, subjective evaluation was strong. Therefore, the graded evaluation method was adopted. The 45 scores of each participant were taken as the mean. The two times data group were respectively named First1 and First 2.

In the second experiment, forty-five students of the same grade in product specialty were organized and these participants are different from those in the first experiment. The number of females is 23 and the male is 22 . In the first stage, every 15 participants randomly are a group, choose one of the following design methods to carry out innovative chair design: Visualization, Analogies, and SCAMPER. The 
three design methods are commonly used in the process of students design products. The contents and rules are the same as the first stage of the first group of experiments in the stage. After four days, the 45 participants were organized to begin the second stage experiment. In the stage, the contents and rules are the same as the second stage of the first group of experiment. The third stage of the experiment is to organize 45 other students majoring product design to score the two design schemes, using the 7point Likert scale. The 45 scores of each participant were taken as the mean. The two times data groups respectively named Second1 and Second2, Visualization and TVC1, Analogies and TVC2, SCAMPER and TVC3.

\section{Results}

In the first experiment, using Kolmogorov-Smirnov to test Free and TVC, the two groups of data were non-normally distributed, $\mathrm{p}<0.001$. Two related samples obtained significant differences in scores between the First1 and Second1 groups $(\mathrm{z}=-3.140, \mathrm{p}=0.002)$, and the scores using TVC were higher than when TVC was not used (Free, median=3.29; TVC, median=4.91). Since the two sets of data are abnormally distributed, we compared them with the median. In the second experiment, statistical analysis of the data is shown in Table 2.

Table 2 Statistical analysis of the data

\begin{tabular}{c|c|c|c}
\hline Data group & Median & Normal distribution & Two related samples test \\
\hline Second1, Second2 & median=3.18, edian=5.07 & $\mathrm{p}<0.001, \mathrm{p}<0.001$ & $\mathrm{z}=-3.324, \mathrm{p}=0.001$ \\
Visualization, TVC1 & median=3.02, edian=5.80 & $\mathrm{p}=0.035, \mathrm{p}<0.001$ & $\mathrm{z}=-1.988, \mathrm{p}=0.047$ \\
Analogies, TVC2 & median=3.84, edian=5.04 & $\mathrm{p}=0.034, \mathrm{p}=0.012$ & $\mathrm{z}=-2.102, \mathrm{p}=0.036$ \\
SCAMPER, TVC3 & median=3.16, edian=5.07 & $\mathrm{p}=0.008, \mathrm{p}=0.004$ & $\mathrm{z}=-2.158, \mathrm{p}=0.031$ \\
\hline
\end{tabular}

The visualized sheet serves as a tool to help students generate product design creativity. On this sheet, as long as the topology variations are manipulated, the hole, inside/outside and a new connectivity are established; a new interaction can be generated between the product and the person, thereby creating a new experience.

\section{Discussion}

The important thing of TVC is that the idea has been created. The characteristic of topological variations is generating ideas first, or making assumptions first, and then demonstrating whether these creativities or assumptions can be achieved. From this aspect, topological variation is prescriptive, reducing human mental work, or moving the human brain activity from generating ideas to demonstrating the feasibility of creativity, reducing the intensity of mental activity. The results from the experiment can be verified, and participants with poor product design ability will have more obvious progress.

In Table 3, the originality of the first sketch of 118 is also in the aspect of morphological transformation. The structure of the chair is the same as that of the normal chair. We can judge that the participant's innovative thinking is normal. It is clear from the second plan that participants broke through the existing logic of thinking and completely broke the stereotype structure of chairs in their own minds. All parts of the chair are fused together, and the chair constantly adjusts its shape according to the sitting position of the person to suit the posture of the person automatically.

Design 222-1 is to combine the shape and storage functions to solve the problem of item placement. Design 222-2 is the child chair. Combine the seat and armrests to expand the way children use them. However, there are certain security risks in this program. The reason may be why design 222-1 is better than design 222-2. Table 4.

Design 201-1 transforms the shape of the chair into a cup that is spilling coffee. It is difficult for people to see this moment in normal times, and this rare form can be used in the shape of a chair, which really brings novelty. The experience of design 201-2 is based on TVC. Applying, trying to change the connection between the various parts of the chair, but the appearance of the chair is quite normal compared to design 201-1, Table 5. 
Table 3. The comparison of sample 118 before and after TVC

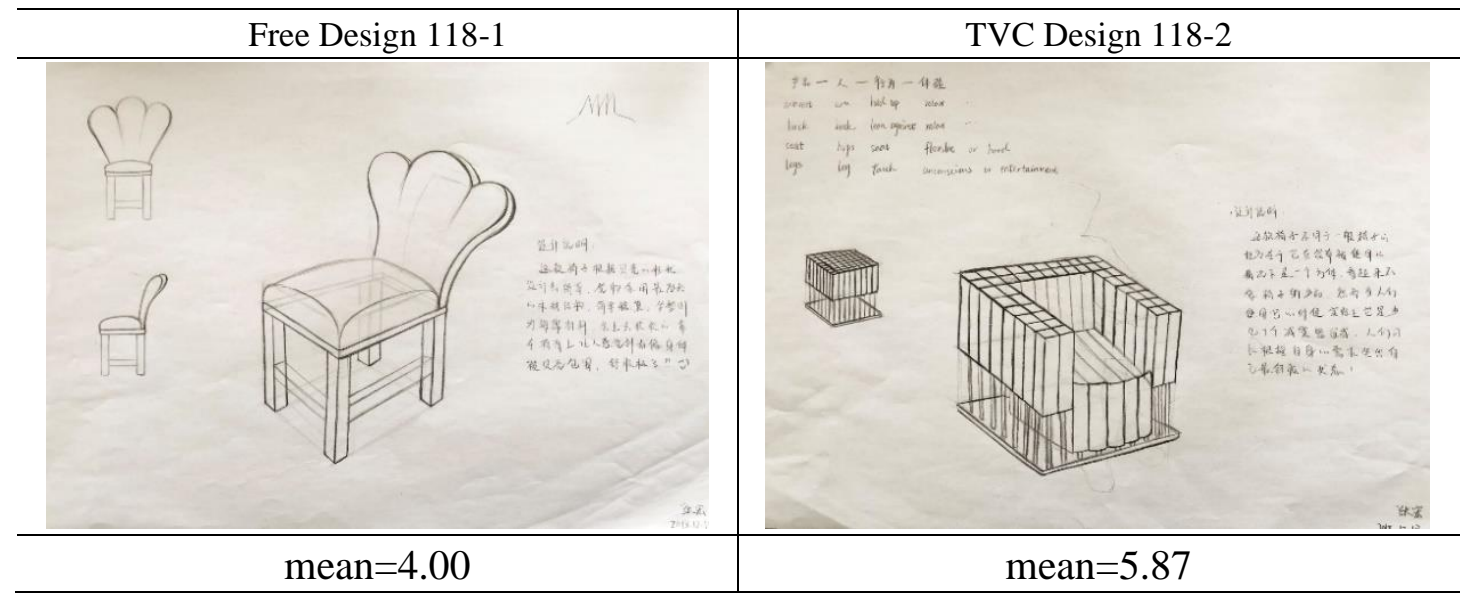

Table 4 The comparison of Visualization and TVC

\begin{tabular}{|c|c|c|c|}
\hline \multicolumn{2}{|r|}{ Visualization Design 222-1 } & \multicolumn{2}{|l|}{ TVC Design $222-2$} \\
\hline 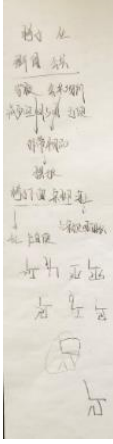 & 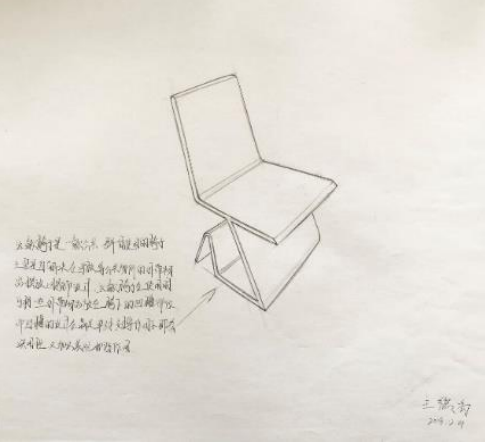 & 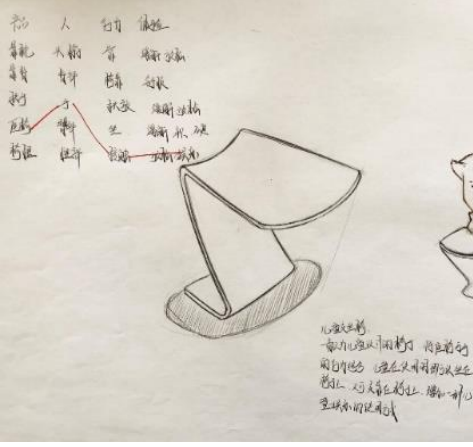 & 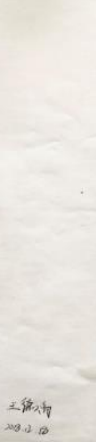 \\
\hline & mean $=5.96$ & mean $=3.93$ & \\
\hline
\end{tabular}

Table 5 The comparison of Analogy and TVC

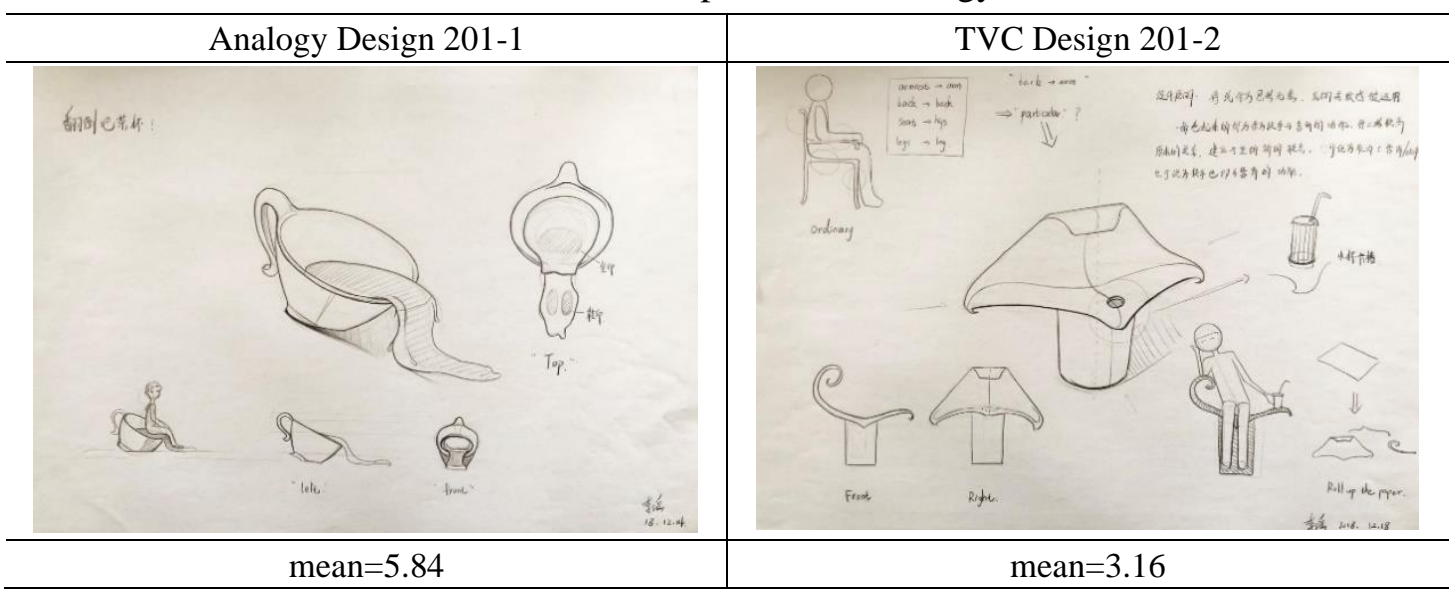

Design 241-1 combines the form of an office chair and a massage chair. There are a lot of office chairs and extra health products designed for office workers on the market, and this kind of design is very mature. Design 241-2 is a seat designed for children's paintings. It is based on children's activity rules and seats for the buttocks and calves, cleverly combining sitting and squatting Table 6 . 
Table 6 The comparison of SCAMPER and TVC

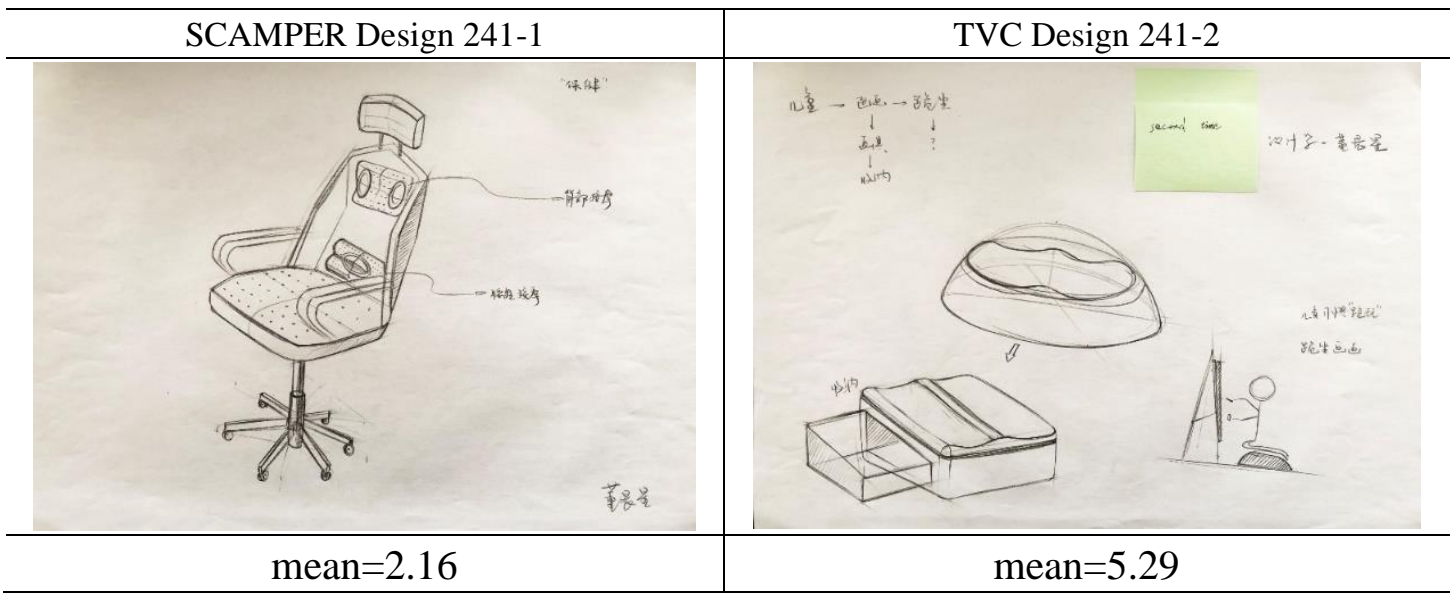

\section{Conclusion}

TVC is currently focusing on the design of individual products, but the reality is that people face product systems rather than individual products. We must consider all the products involved in the specified range. In this case, the single visual sheet is obviously not suitable for the production system design. Future research efforts will focus on the application of topological variability in product system design. In the information society, human beings yearn for the harmonious coexistence of a man-machine environment that requires designers not only to consider the functional needs but also to consider the emotional language of products, and whether they can achieve the purpose of emotional appeal (Nagai, 2015). If consumers can find psychological resonance in products and have a pleasant attitude, this is the embodiment of an excellent product. Future research will apply the model to a wider range of creative activities, not limited to the product form design. Using the approach, it can be extended to other event processing, such as information products design.

\section{References}

Chen, L. (2005). The topological approach to perceptual organization. Visual Cognition, 12(4), 553-637.

Chen, L. (1982). Topological structure in visual perception. Science, 218(4573), 699-700.

Darke, I. (1982). A review of research related to the topological primacy thesis. Educational Studies in Mathematics, 13(2), 119-142.

Fei, F., \& Nagai, Y. (2019). Topological perception on attention to product shape. International Journal of Design Creativity and Innovation, 1-14.

Hekkert, P., Snelders, D., Van Wieringen, P.C.W., 2003. 'Most advanced, yet acceptable': typicality and novelty as joint predictors of aesthetic preference in industrial design. Br. J. Psychol. 94, 111-124.

Hollins, B., and S. Pugh. 1990. Successful product design: What to do and when. London: Butterworth.

Munkres, J. (2014). Topology. Pearson Education.

Nagai, Y. (2015). A sense of design: The embedded motives of nature, culture, and future. In Principia DesignaePre-Design, Design, and Post-Design (pp. 43-59). Springer, Tokyo.

Navon, D. (1977). Forest before trees: The precedence of global features in visual perception. Cognitive psychology, 9(3), 353-383.

Rashevsky, N. (1954). Topology and life: in search of general mathematical principles in biology and sociology. The bulletin of mathematical biophysics, 16(4), 317-348.

Rindova, V., and A. Petkova. 2007. When is a new thing a good thing? Technological change, product form design, and perceptions of value for product innovations. Organization Science 18 (2): 217-32.

Ruth Mugge, Jan P.L. Schoormans (2012). Product design and apparent usability. The influence of novelty in product appearance. Applied Ergonomics, 43, 1081-1088.

Treisman, A. M., \& Gelade, G. (1980). A feature-integration theory of attention. Cognitive psychology, 12(1), 97-136. 